CLINICAL STUDY

\title{
Blockade of oestrogen biosynthesis in peripubertal boys: effects on lipid metabolism, insulin sensitivity, and body composition
}

\author{
Matti Hero ${ }^{1}$, Carina Ankarberg-Lindgren ${ }^{3}$, Marja-Riitta Taskinen ${ }^{2}$ and Leo Dunkel ${ }^{4}$ \\ ${ }^{1}$ Hospital for Children and Adolescents and ${ }^{2}$ Department of Medicine, University of Helsinki and Helsinki University Central Hospital, Helsinki, Finland, \\ ${ }^{3}$ Göteborg Paediatric Growth Research Center, Institute for the Health of Women and Children, Göteborg University, Göteborg, Sweden and ${ }^{4}$ Department of \\ Paediatrics, Kuopio University Hospital, Kuopio, Finland \\ (Correspondence should be addressed to L Dunkel; Email: leo.dunkel@kuh.fi)
}

\begin{abstract}
Objective: In males, the pubertal increase in sex hormone production has been associated with proatherogenic changes in lipid and carbohydrate metabolism. Aromatase inhibitors, a novel treatment modality for some growth disorders, may significantly influence these risk factors for cardiovascular disease by suppressing oestrogen biosynthesis and stimulating gonadal androgen production. In the current study, we explored the effects of aromatase inhibition on lipid metabolism, insulin sensitivity, body composition and serum adiponectin in peripubertal boys.

Design: Prospective, double-blind, randomised, placebo-controlled clinical study.

Methods: Thirty-one boys, aged 9.0-14.5 years, with idiopathic short stature were treated with the aromatase inhibitor letrozole $(2.5 \mathrm{mg} / \mathrm{day})$ or placebo for 2 years. During the treatment, the concentrations of sex hormones, IGF-I, lipids, lipoproteins and adiponectin were followed-up. The percentage of fat mass (FM) was assessed by skinfold measurements and insulin resistance by homeostasis model assessment (HOMA) index.

Results: In pubertal boys, who received letrozole, high-density lipoprotein cholesterol (HDL-C) decreased by $0.47 \mathrm{mmol} / \mathrm{l}(P<0.01)$ during the study. Simultaneously, their percentage of FM decreased from 17.0 to $10.5(P<0.001)$, in an inverse relationship with serum testosterone. The concentrations of low-density lipoprotein cholesterol, triglycerides and HOMA index remained at pretreatment level in both groups. Serum adiponectin decreased similarly in letrozole- and placebotreated pubertal boys ( 2.9 and $3.3 \mathrm{mg} / \mathrm{l}$ respectively).

Conclusions: In males, aromatase inhibition reduces HDL-C and decreases relative FM after the start of puberty. The treatment does not adversely affect insulin sensitivity in lean subjects.
\end{abstract}

European Journal of Endocrinology 155 453-460

\section{Introduction}

During male puberty, concomitant with hormonal and physical changes, lipid and carbohydrate metabolism changes to a proatherogenic direction. In particular, high-density lipoprotein cholesterol (HDL-C) concentration and insulin sensitivity decrease (1-3). This reduction in HDL-C has been attributed mainly to a coinciding increase in testosterone secretion (4), while the transient development of insulin resistance appears to be more dependent on augmented growth hormone (GH) secretion $(2,5)$.

Pubertal hormonal changes are strongly modulated by aromatase inhibitors. By reducing oestrogen biosynthesis, they delay bone maturation; therefore, these compounds have been used in the treatment of various growth disorders such as familial male limited precocious puberty (6), congenital adrenal hyperplasia (7), McCune-Albright syndrome (8), constitutional delay of growth and puberty (9) and idiopathic short stature
(ISS) (10). Suppression of oestrogen biosynthesis is followed by an increase in gonadotrophin and gonadal androgen secretion in pubertal boys (11), and attenuation of the pubertal oestrogen-dependent stimulation of the GH-insulin-like growth factor I system $(9,10)$. Thus, in males during puberty, aromatase inhibitors could decrease HDL-C and improve insulin sensitivity (12). However, findings in men with congenital oestrogen deficiency suggest that prolonged lack of oestrogen effects, in the presence of normal or high testosterone concentration, predisposes both to impaired lipid profile and insulin resistance at an early age (13-15). Inhibition of aromatase activity in males may thus predispose to features of metabolic syndrome. The effects of aromatase inhibition on lipid and carbohydrate metabolism during adolescence have been inadequately characterised.

In the current prospective, randomised, double-blind trial, we studied the effects of aromatase inhibition on lipid metabolism, insulin sensitivity and body 
composition in boys during prepuberty and progression of puberty. In addition, we investigated whether modulation of the sex hormone environment by aromatase inhibition influences the adipocytokine adiponectin, low levels of which are associated with insulin resistance (16), reduced HDL-C level (17) and signs of early atherosclerosis (18). The study cohort included 31 healthy initially peri- or early-pubertal boys with ISS, who were treated with the aromatase inhibitor letrozole (Lz), or placebo $(\mathrm{Pl})$, for 2 years in an attempt to increase adult height.

\section{Materials and methods}

\section{Subjects}

As previously described (10), the study population comprised short boys examined and followed-up for ISS at the outpatient clinic for paediatric endocrinology at the Hospital for Children and Adolescents, University of Helsinki, Finland. Inclusion criteria for the study were: calendar age of 9.0-14.5 years and height at least 2 s.D. below the mean for age or at least 2 s.D. below midparental target height. Only boys with a bone age of 14 years or less were recruited. GH deficiency was ruled out by a GH stimulation test for those with slow growth velocity or subnormal serum insulin-like growth factor-I (IGF-I) or IGF binding protein 3 (IGFBP-3) concentrations. Between May 2001 and May 2002, 31 boys with ISS were enrolled, but after 6 months of treatment one boy receiving $\mathrm{Pl}$ was diagnosed with diabetes mellitus and excluded. During the study period, none of the patients received any medication, other than Lz, known to affect lipid or carbohydrate metabolism.

\section{Study design}

The boys were randomised in a double-blind manner to receive either Lz (Femar, Novartis AG), $2.5 \mathrm{mg}$ orally once daily, or Pl orally once daily for 2 years. The investigators and the subjects were blinded to treatment assignment until the end of follow-up. The boys were examined at entry and every 6 months thereafter during the 2-year treatment. Follow-up visits included a physical examination, performed by the same physician (M Hero) on all the occasions. Stage of puberty was evaluated according to Tanner (19). Height was measured on a Harpenden stadiometre with $0.1 \mathrm{~cm}$ precision. The amount of fat mass (FM) relative to total body mass (percentage of FM) was evaluated by six skinfold measurements, as previously described (20). A measure of insulin resistance, the homeostasis model assessment (HOMA) index, was calculated as follows: HOMA index $=($ fasting insulin $(\mathrm{mU} / \mathrm{l}) \times$ fasting glucose $(\mathrm{mmol} / \mathrm{l})) / 22.5(21)$.

In a previous study, we showed that, in pubertal boys, Lz treatment enhances gonadotrophin secretion by reducing the level of oestrogen-mediated negative feedback (11). Consequently, Lz expedites testicular growth and enhances testosterone secretion after the onset of puberty. As previously reported (10), this was also apparent in the pubertal boys of the current study (Fig. 1A). Augmented testosterone secretion in Lz-treated boys resulted in slightly faster progression of puberty compared to that of Pl-treated boys, as suggested by higher Tanner pubic hair $(\mathrm{P})$ stage of puberty at 12 $(P<0.05), 18(P<0.05)$ and 24 months $(P=0.06)$ in those treated with $\mathrm{Lz}$ (Table 1). No significant differences in Tanner genital (G) stages of puberty appeared between the treatment groups during follow-up. Despite the more rapid progression of puberty, the puberty-associated increase in IGF-I concentration in pubertal Lz-treated boys was inhibited (Fig. 1C), most probably reflecting decreased GH secretion.
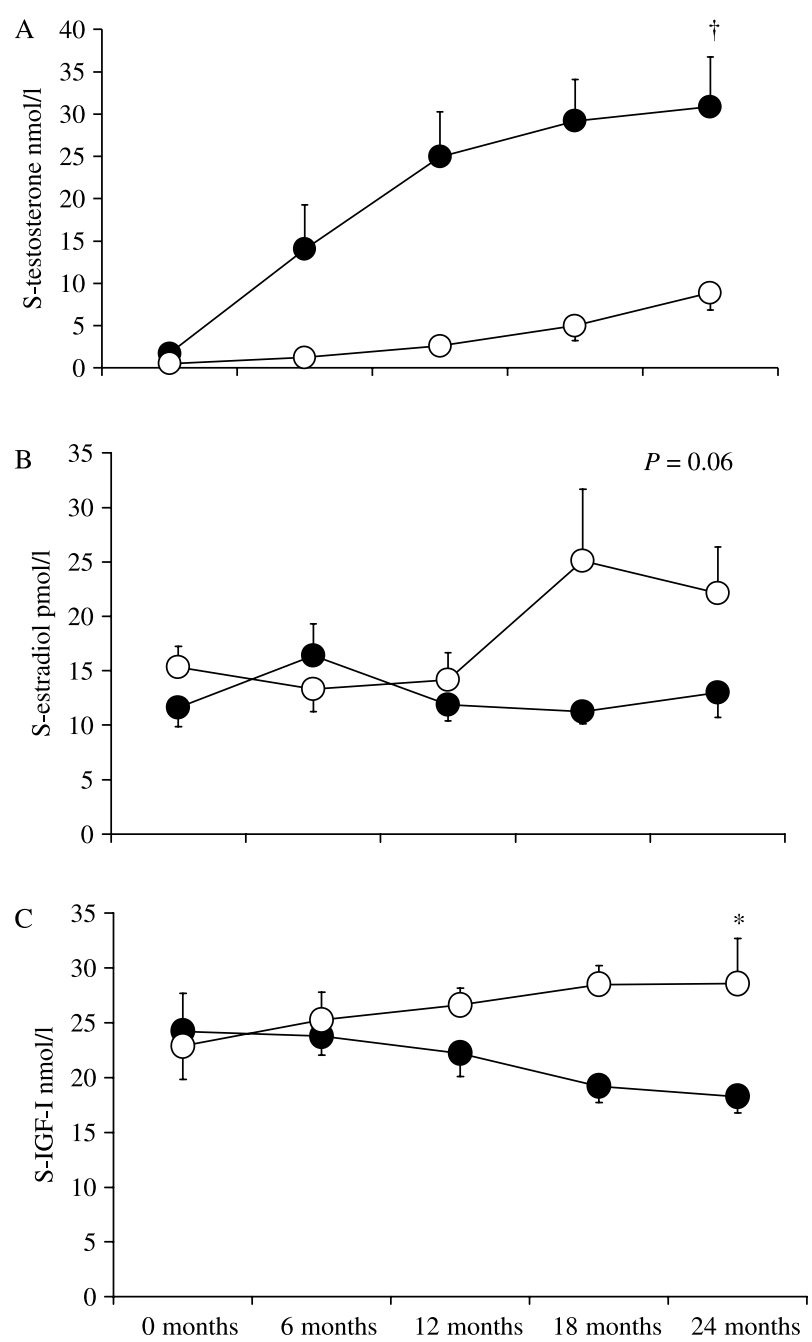

Figure 1 Concentrations of serum testosterone, oestradiol, and IGF-I in letrozole- $(\bullet, n=9)$, and placebo-treated $(\bigcirc, n=8)$, pubertal boys. Values are mean (s.E.M.). ${ }^{\star} P<0.05$ and ${ }^{\dagger} P<0.01$ refer to difference between groups as evaluated by repeated measures ANOVA, with treatment as a between-subjects factor. 
Table 1 Baseline characteristics and stage of puberty after 2 years of treatment, in the prepubertal and pubertal subgroups of boys with idiopathic short stature (ISS) randomised to receive letrozole (Lz) or placebo. Those remaining prepubertal throughout follow-up were classified as prepubertal; those entering puberty within 18 months after the start of follow-up were classified as pubertal. Values are mean (S.D.), except for Tanner genital (G) and pubic hair (P) stages of puberty, which are medians (range).

\begin{tabular}{|c|c|c|c|c|}
\hline & \multicolumn{2}{|c|}{ Prepubertal boys } & \multicolumn{2}{|c|}{ Pubertal boys } \\
\hline & $\begin{array}{c}\mathrm{Lz} \\
(n=7)\end{array}$ & $\begin{array}{l}\text { Placebo } \\
(n=6)\end{array}$ & $\begin{array}{c}\mathrm{Lz} \\
(n=9)\end{array}$ & $\begin{array}{c}\text { Placebo } \\
(n=8)\end{array}$ \\
\hline Age (years) & $9.5(0.7)$ & $10.3(0.8)$ & $12.2(1.1)$ & $11.5(1.7)$ \\
\hline Height $(\mathrm{cm})$ & $122.0(1.7)$ & $124.3(3.4)$ & $133.6(4.6)$ & $130.1(8.0)$ \\
\hline Weight (kg) & $24.2(4.0)$ & $23.9(1.9)$ & $30.8(4.3)$ & $27.6(4.9)$ \\
\hline BMI $\left(\mathrm{kg} / \mathrm{m}^{2}\right)$ & $16.3(2.5)$ & $15.4(0.7)$ & $17.2(1.7)$ & $16.2(1.5)$ \\
\hline Body fat (\%) & $14.6(6.9)$ & $14.3(2.8)$ & $17.7(4.9)$ & $14.2(3.2)$ \\
\hline G-stage at start & 1 & 1 & $1(1-3)$ & $1(1-2)$ \\
\hline At 24 months & 1 & 1 & $4(2-5)$ & $3(2-4)$ \\
\hline P-stage at start & 1 & 1 & $1(1-2)$ & $1(1)$ \\
\hline At 24 months & 1 & 1 & $4(1-5)$ & $2(1-4)$ \\
\hline $\begin{array}{l}\text { Testis volume } \\
\text { (ml) }\end{array}$ & $0.7(0.2)$ & $0.7(0.4)$ & $1.8(1.3)$ & $1.0(0.5)$ \\
\hline
\end{tabular}

BMI, body mass index.

Due to the divergent effects of $\mathrm{Lz}$ on testosterone secretion before and after the onset of puberty, we also wanted to analyse the treatment effect separately in prepubertal and pubertal boys. Therefore, the boys were further subdivided into two groups: those who remained prepubertal (testis volume $\leq 2 \mathrm{ml}$ ) throughout the followup, and those who entered puberty (testis volume $>2 \mathrm{ml}$ ) within 18 months after the start of treatment. According to these criteria, 7 of 16 and 6 of 14 boys remained prepubertal, and 9 of 16 and 8 of 14 progressed into puberty in the Lz and Pl groups respectively.

The study protocol was approved by the Ethics Committee of the Hospital for Children and Adolescents, and by the National Agency for Medicines. Before the initiation of treatment, written informed consent was obtained from the boy and his guardian(s).

\section{Biochemical measurements}

Venous blood samples were drawn between 0730 and $1000 \mathrm{~h}$. Concentrations of lipids and IGF-I were determined directly after each follow-up visit, and the concentrations of testosterone and oestradiol from sera stored at -20 or $-70{ }^{\circ} \mathrm{C}$ until required. Serum testosterone concentrations were determined with a modified RIA (Spectria Testosterone RIA, Orion Diagnostica) accredited by Swedac (no 1899), as described previously (22). Serum oestradiol concentrations were quantified with an accredited (as above) modified RIA (Spectria E2, ORION Diagnostica, Espoo, Finland) with a detection limit of $4.5 \mathrm{pmol} / \mathrm{l}$, as described perviously (23). Concentrations of serum IGF-I were quantitated with RIA (DiaSorin, Stillwater, Minnesota, USA). Serum total cholesterol and triglycerides were determined by enzymatic methods (Hoffman-La Roche kits 0722138 and 0715166 Basel, Switzerland). Serum HDL-C and subfractions were quantified by phosphotungastic acid/ magnesium chloride precipitation procedures (Hoffman-La Roche kit 0720674). Serum low-density lipoprotein cholesterol (LDL-C) was calculated by the Friedewald formula (24). Concentrations of apolipoprotein AI (Apo AI), apolipoprotein AII (Apo AII) and apolipoprotein B (Apo B) were measured by immunoturbidometric methods with commercial kits (Boehringer-Mannheim, Mannheim, Germany). Serum insulin concentrations were measured with a fluoroimmunometric assay (AutoDelfia Insulin, Wallac, Turku, Finland) and serum adiponectin concentrations with a sandwich ELISA-based assay (Human Adiponectin ELISA Kit, B-Bridge International, Inc., San Jose, USA). Lipoprotein (a) (Lp(a)) levels were determined with a turbidimetric immunoassay (Lp(a)-HA WAKO Chemicals $\mathrm{GmbH}$, Neuss, Germany). Blood glucose values were quantified with amperometric glucose oxidase method (EBIO Compact, Eppendorf, Hamburg, Germany).

\section{Statistical analysis}

Two-sided tests of hypotheses were used and a $P$-value $<$ 0.05 was considered significant. Values are expressed as mean \pm s.D., unless otherwise stated. The analyses were conducted with SPSS statistical software for Windows, release 10.0 (SPSS, Inc., Chicago, Illinois, USA). Betweengroup differences in baseline characteristics were evaluated by the Mann-Whitney U-test (Tanner stages) and the $t$-test (others). In pubertal boys, within-group changes in weight, in body mass index (BMI), in percentage of FM, and in biochemical markers were analysed by repeated measures ANOVA, using log-transformed data when appropriate. In prepubertal boys, the Wilcoxon signed ranks test was employed to analyse changes in biochemical parameters and body composition. Between-group differences in pubertal stages were analysed by the Mann-Whitney U-test. Repeated measures ANOVA was used to analyse the interactions between received treatment $(\mathrm{Lz} / \mathrm{Pl})$ and lipids, treatment and HOMA index, stage of puberty (prepubertal/pubertal) and lipids, and stage of puberty and HOMA index. The effects of BMI, percentage of FM, testosterone, oestradiol, IGF-I and adiponectin on HDL-C and HOMA index during the treatment were evaluated with repeated measures ANOVA with 2-year changes in the variables mentioned above as covariates. Spearman correlation coefficient was used in analysis of correlations.

\section{Results}

At baseline, no significant differences between treatment groups appeared in age, weight, BMI, percentage of FM or pubertal maturation (Table 1). 


\section{Lipids and lipoproteins}

In Lz-treated boys, a significant decrease in HDL2-C (from 0.50 to $0.32 \mathrm{mmol} / \mathrm{l}, P<0.01$ ), Apo AI (from 140 to $116 \mathrm{mg} / \mathrm{dl}, P<0.001$ ) and Apo AII (from 40.1 to $32.0 \mathrm{mg} / \mathrm{dl}, P<0.01)$ occurred during the study. Similarly, HDL2-C (from 0.62 to $0.50, P=0.09$ ), Apo AI (from 146 to $129 \mathrm{mg} / \mathrm{dl}, P<0.01$ ) and Apo AII (from 39.7 to $31.4 \mathrm{mg} / \mathrm{dl}, \mathrm{P}<0.001$ ) decreased in boys receiving $\mathrm{Pl}$. No changes in other lipids or lipoproteins were evident. Including all patients in the analysis, no significant interactions between received treatment and lipids or lipoproteins were found. Instead, an interaction between HDL-C and puberty $(P<0.05)$, and between Lp(a) and puberty $(P<0.05)$ was detected. Given that changes in concentrations of sex hormones occurred only in boys entering puberty during the study period (Fig. 1), we next evaluated the effects of the treatment in the subgroup of pubertal subjects.

In pubertal boys receiving Lz, HDL-C decreased by $0.47 \mathrm{mmol} / \mathrm{l}(P<0.01)$ in 2 years (Fig. $2 \mathrm{~A})$, a reduction particularly apparent in the HDL2-subclass (Fig. 2B); HDL-C of the Pl-treated pubertal boys did not change significantly. A significant interaction existed between HDL-C and received treatment $(P<0.05)$. Concomitant to decrease in HDL-C, Apo AI concentration decreased markedly in Lz-treated pubertal boys, but did not change significantly in the Pl-treated boys (Fig. 2D) (mean decreases 38 vs $13 \mathrm{mg} / \mathrm{dl}$ respectively, $P<0.0001)$. Apo AII concentration decreased in pubertal boys of both groups (Fig. 2E), and the magnitude of the change was similar (10 vs $9 \mathrm{mg} / \mathrm{dl}$ respectively). Concentrations of total cholesterol, LDL-C, Apo B and triglycerides remained at pretreatment level throughout the study period in pubertal boys of both groups (Table 2). Lp(a) decreased only in Lz-treated pubertal boys (Table 2).

The 2-year changes in BMI, percentage of FM, testosterone, oestradiol and adiponectin were selected as potential predictors of lipid concentrations during treatment. Among Lz-treated subjects, including both prepubertal and pubertal boys, these predictors were interrelated: a strong negative correlation existed between change in testosterone and change in percentage of FM $(r=-0.76, P<0.01)$, and a positive correlation between change in adiponectin and change in percentage of FM $(r=0.60, P<0.05)$. In addition, change in testosterone showed a negative correlation with change in adiponectin, which failed to reach significance $(r=-0.47, P=0.07)$. We next evaluated the contribution of each predictor to the regulation of HDL-C with repeated measures ANOVA, using one predictor at a time as a covariate. Among Lz-treated boys, a significant interaction occurred between change in percentage of $\mathrm{FM}$ and $\mathrm{HDL}-\mathrm{C}$ during treatment $(P<0.01)$, and between change in testosterone and HDL-C $(P=0.01)$. In addition, the change in adiponectin showed a borderline interaction with HDL-C $(P=0.05)$. Correspondingly, the changes in percentage of FM $(r=0.67, \quad P<0.01), \quad$ testosterone $\quad(r=-0.55$, $P<0.05)$, and adiponectin $(r=0.51, P<0.05)$ all correlated significantly with the treatment-induced change in HDL-C; the unexpected positive correlation between changes in percentage of FM and HDL-C may reflect a suppressing effect of testosterone on both factors. In line with findings in HDL-C, the changes in percentage of FM $(r=0.82, P<0.001)$, testosterone $(r=-0.56$, $P<0.05)$, and adiponectin $(r=0.55, P<0.05)$ all correlated with the treatment-induced change in Apo AI. Changes in oestradiol and BMI, as evaluated by repeated measures ANOVA, had no significant influence on HDL-C level during follow-up.

No significant interactions between LDL-C level and the selected predictors (2-year changes in BMI, percentage of FM, testosterone, oestradiol or adiponectin) appeared in Lz-treated boys. Similarly, no interactions appeared between triglyceride concentration during treatment and the above-mentioned predictors. However, the change in testosterone correlated negatively with the change in Lp(a) in Lz-treated boys ( $r=-0.86, P<0.01)$.

\section{Body composition and carbohydrate metabolism}

Including all subjects in the analysis, no significant changes in percentage of FM were detected, whereas BMI increased by $1.8(P<0.0001)$ and $1.5 \mathrm{~kg} / \mathrm{m}^{2}$ $(P<0.0001)$ during the treatment in $\mathrm{Lz}$ and $\mathrm{Pl}$ groups respectively. No interaction occurred between BMI and received treatment or between percentage of FM and received treatment. HOMA index remained at pretreatment level during the study in both groups. Further, no interaction between HOMA index and received treatment or between HOMA index and pubertal maturation was found.

We next analysed the effects of the treatment in pubertal boys. Weight and BMI increased similarly in Lz- and Pl-treated pubertal subjects, whereas the percentage of FM decreased only in those who received $\mathrm{Lz}$ (Table 3). No significant changes in concentrations of fasting blood glucose, insulin or in HOMA index occurred during treatment in either group (Table 3). Moreover, no blood glucose or insulin values above normal were evident in any patient. No significant interaction appeared between treatment $(\mathrm{Lz} / \mathrm{Pl})$ and HOMA index or insulin level, as analysed by repeated measures ANOVA. Using the 2-year changes in BMI in percentage of FM, testosterone, oestradiol, IGF-I and adiponectin as covariates, no interactions with HOMA index were detected $(P=0.16-0.62)$. Serum adiponectin level decreased similarly in Lz- and Pl-receiving pubertal boys during the study period (Table 3; 2.9 and $3.3 \mathrm{mg} / \mathrm{l}$ respectively).

Serum alanine aminotransferase concentrations remained at their pretreatment levels in both treatment groups (data not shown), and no abnormal values were evident in any patient at any point. 

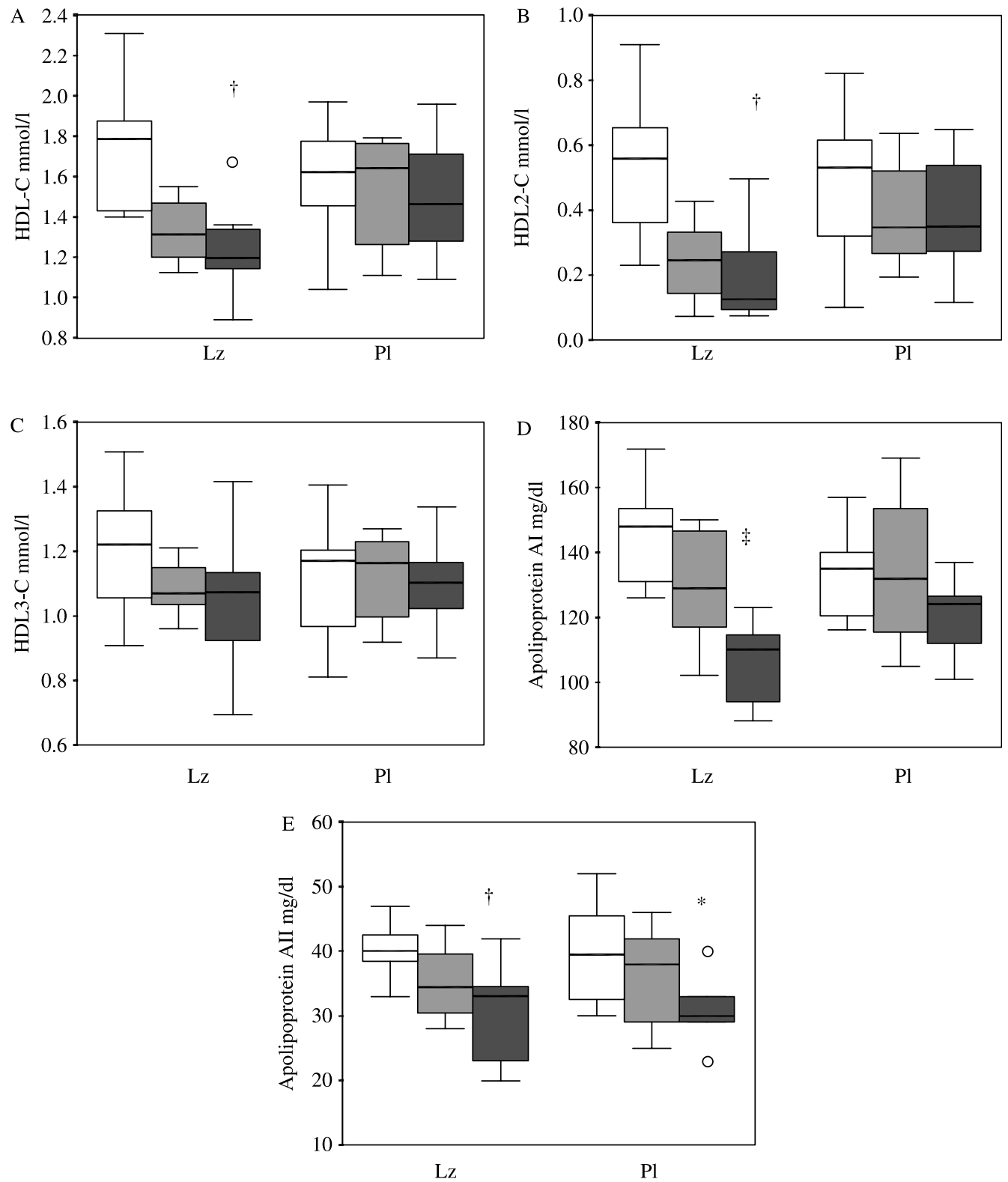

Figure 2 Response of high-density lipoprotein cholesterol (HDL-C) and apolipoproteins in pubertal letrozole- (Lz, $n=9$ ) and placebo-treated $(\mathrm{PI}, n=8)$ boys. Boxes represent interquartile ranges $(50 \%$ of values) with medians. Whiskers represent total ranges, excluding outliers (circles). ${ }^{\star} P<0.05$ and ${ }^{\dagger} P<0.01$ refer to change from baseline evaluated by repeated measures ANOVA. HDL2-C, HDL2-cholesterol; HDL3-C, HDL3-cholesterol. White boxes represent 0 month values, light grey boxes 12 month values, dark grey boxes 24 month values.

\section{Prepubertal boys}

Boys who remained prepubertal throughout the study showed no changes in concentrations of sex hormones (data not shown). In prepubertal boys treated with Lz, total cholesterol increased slightly during the 2 years of treatment (from 4.0 to $4.5 \mathrm{mmol} / \mathrm{l}, P<0.05$ ), with no significant changes in LDL-C (from 2.2 to $2.5 \mathrm{mmol} / \mathrm{l}$ ) or HDL-C (from 1.6 to $1.8 \mathrm{mmol} / \mathrm{l}$ ). Simultaneously, their BMI increased from 16.3 to $17.8 \mathrm{~kg} / \mathrm{m}^{2}(P<0.05)$ and percentage of FM from 14.6 to $18.6(P<0.05)$, whereas no changes were apparent in HOMA index (0.8 and 1.0 at 0 and 24 months), or adiponectin (11.0 and $11.3 \mathrm{mg} / \mathrm{l}$ at 0 and 24 months). In prepubertal Pltreated boys, cholesterol, HOMA index and adiponectin levels remained unchanged, with a slight increase in BMI (data not shown).

\section{Discussion}

In males, after the onset of puberty, treatment with the aromatase inhibitor Lz produces a hormonal milieu 
Table 2 Changes in total cholesterol, LDL-cholesterol (LDL-C), triglycerides (TG), apolipoprotein B (Apo B), and lipoprotein (a) (Lp(a)) in pubertal boys treated with letrozole (Lz) or placebo. Values are mean (s.D.), except for values of Apo B and $L p(a)$, which are geometric means due to skewed distributions.

\begin{tabular}{|c|c|c|c|c|c|c|}
\hline & \multicolumn{3}{|c|}{ Lz; months } & \multicolumn{3}{|c|}{ Placebo; months } \\
\hline & 0 & 12 & 24 & 0 & 12 & 24 \\
\hline Total cholesterol $(\mathrm{mmol} / \mathrm{l})$ & $4.6(1.0)$ & $4.3(0.8)$ & $4.4(1.1)$ & $4.3(0.4)$ & $4.3(0.5)$ & $4.3(0.3)$ \\
\hline LDL-C (mmol/l) & $2.6(0.9)$ & $2.7(0.8)$ & $2.8(1.0)$ & $2.4(0.4)$ & $2.4(0.4)$ & $2.5(0.5)$ \\
\hline $\mathrm{TG}(\mathrm{mmol} / \mathrm{l})$ & $0.7(0.2)$ & $0.6(0.2)$ & $0.7(0.3)$ & $0.8(0.2)$ & $0.7(0.2)$ & $0.6(0.2)$ \\
\hline Apo B (mg/dl) & 70.8 & 75.9 & 77.6 & 74.1 & 74.1 & 75.9 \\
\hline $\mathrm{Lp}(\mathrm{a})(\mathrm{mg} / \mathrm{l})$ & 104.7 & 114.8 & $95.5^{\star}$ & 89.1 & 89.1 & 104.7 \\
\hline
\end{tabular}

${ }^{\star} P<0.05$ compared with baseline value (Student's paired $t$-test after log-transformation).

characterised by high testosterone and low oestradiol concentrations, and a clinically significant reduction in HDL-C level. This finding is in accord with our recent findings on Lz treatment in boys with delayed puberty, in whom Lz reduced HDL-C in an inverse relationship with testosterone (12). Studies with another thirdgeneration aromatase inhibitor, anastrozole, have reported conflicting results. Anastrozole does not appear to influence lipid profile, fasting blood glucose or insulin levels in healthy (25) or GH-deficient (26) adolescent boys. The reason for these differing findings with the two compounds is unclear, but this may reflect the greater potency of Lz to inhibit oestrogen synthesis and consequently, to increase androgen secretion (27).

In our Lz-treated boys, the change in HDL-C was negatively associated with change in testosterone, and was positively associated with change in adiponectin, supporting a role for these hormones in regulation of HDL-C during puberty. In support of the role of testosterone, androgens are known to enhance HDL-C catabolism by increasing hepatic lipase activity, especially during simultaneous suppression of oestrogen production (28). This would also explain why Lz treatment influenced HDL2-C and Apo AI concentrations in particular, because large HDL species are the preferred substrates of hepatic lipase $(28,29)$. Oestrogen suppression probably did not contribute to our subjects' reduction in HDL-C, since the concentration of oestradiol was not associated with the fall in HDL-C in Lz-treated boys. Furthermore, the change in testosterone: oestradiol ratio was not more strongly correlated with the change in HDL-C than the change in testosterone was (data not shown).

The positive association between change in adiponectin and change in HDL-C in Lz-treated boys suggests that also adiponectin contributes to the regulation of HDL-C level in pubertal males. Indeed, studies show that adiponectin is positively associated with HDL-C in nondiabetic individuals (30), patients with type-2 diabetes (31) and, according to a recent report, in obese children and adolescents (32). This association between adiponectin and HDL-C has typically remained after controlling for differences in BMI and measures of FM. However, the mechanism by which adiponectin may influence HDL-C is largely unknown. One potential mechanism is that adiponectin directly enhances the synthesis of Apo AI via stimulation of PPAR $\alpha$ expression (16). Alternatively, low adiponectin may enhance HDLC catabolism by stimulating hepatic lipase (33).

The concentrations of LDL-C, Apo B and triglycerides did not change in our pubertal boys of either treatment group during the study period, despite the marked increase in testosterone concentration in Lz-treated boys, and increases in both testosterone and oestradiol in Pl-treated boys. This finding strongly argues against significant sex hormone-related regulation of LDL-C and triglycerides in boys during early- and mid-puberty, and corresponds to the findings in other studies of aromatase inhibitor treatment in males during adolescence $(12,25,26)$. However, concentrations of $\operatorname{Lp}(\mathrm{a})$

Table 3 Changes in body composition and markers of carbohydrate metabolism in pubertal boys treated with letrozole (Lz) or placebo. Values are mean (S.D.), except for values of weight, percentage of fat mass, serum insulin, and homeostasis model assessment (HOMA) index, which are geometric means due to skewed distributions.

\begin{tabular}{|c|c|c|c|c|c|c|}
\hline & \multicolumn{3}{|c|}{ Lz; months } & \multicolumn{3}{|c|}{ Placebo; months } \\
\hline & 0 & 12 & 24 & 0 & 12 & 24 \\
\hline Weight (kg) & 30.9 & 34.7 & $40.7 \dagger$ & 26.9 & 30.9 & $35.5 \dagger$ \\
\hline $\operatorname{BMI}\left(\mathrm{kg} / \mathrm{m}^{2}\right)$ & $16.8(1.7)$ & $17.5(1.8)$ & $18.7(2.0) \dagger$ & $16.3(1.5)$ & $16.9(1.9)$ & $17.8(2.0) \dagger$ \\
\hline Percentage of fat mass (\%) & 17.0 & 12.3 & $10.5 t$ & 14.1 & 16.6 & 14.5 \\
\hline B-glucose $(\mathrm{mmol} / \mathrm{l})$ & $4.7(0.5)$ & $4.7(0.3)$ & $4.6(0.3)$ & $4.7(0.3)$ & $4.8(0.5)$ & $4.9(0.3)$ \\
\hline S-insulin (mU/l) & 4.7 & 4.1 & 4.2 & 4.2 & 3.9 & 5.8 \\
\hline HOMA index & 0.98 & 0.85 & 0.86 & 0.88 & 0.82 & 1.25 \\
\hline S-adiponectin (mg/l) & $10.9(3.6)$ & $8.6(3.1)$ & $8.0(2.7)^{\star}$ & $11.2(4.2)$ & $10.0(4.1)$ & $7.9(2.7) \dagger$ \\
\hline
\end{tabular}

${ }^{*} P<0.05, \dagger P<0.001$ compared with baseline value. Repeated measures ANOVA was employed for statistical analysis. 
decreased in pubertal boys during treatment with Lz, and the change in $\mathrm{Lp}(\mathrm{a})$ showed a strong negative correlation with the change in testosterone. Since the concentrations of oestradiol and IGF-I remained unchanged, this finding supports the view that, in adolescent males, testosterone reduces $\mathrm{Lp}$ (a) concentration directly, not by its conversion to oestrogen. Further supporting this concept, administration of testosterone to adult males appears to reduce $\mathrm{Lp}(\mathrm{a})$, independently of oestrogen (34). Since $L p(a)$ level is considered an independent risk factor for coronary heart disease (35), this negative association between testosterone and Lp(a) suggests that androgens also have direct beneficial effects on lipid metabolism during puberty.

In pubertal boys, Lz treatment was associated with several metabolic and physical changes that potentially influence insulin sensitivity: serum testosterone increased substantially; relative FM decreased markedly in an inverse relationship with testosterone; and suppression of GH secretion occurred, as suggested by the opposite changes in IGF-I in the Lz- and Pl-groups. The net effect of these changes on carbohydrate metabolism may be positive, since insulin resistance, assessed by HOMA index, remained unchanged in Lz-treated pubertal boys despite progression of puberty from median genital stages 1 to 4 during the treatment. Although differences between pubertal Lz- and Pl-treated boys in HOMA index were not detected, our findings are in line with previous findings on Lz treatment in boys with delayed puberty, in whom serum fasting insulin level decreased during treatment with $\mathrm{Lz}$ but not with Pl (12). Together, these studies indicate that direct androgen effects do not contribute to development of insulin resistance in males during puberty. Instead, aromatisation of androgens to oestrogens and the subsequent stimulation of the GH-IGF-I system probably play a fundamental role. This concept is supported by the close connection between the rise and fall in insulin resistance assessed by the euglycemic hyperinsulinemic clamp technique, and the rise and fall in serum IGF-I level during puberty (5). In our study, IGF-I as well as HOMA index remained at pretreatment levels during follow-up in Lz-treated subjects, who progressed in puberty, but no correlations appeared between these two measures.

Recent studies have indicated that the secretion of adiponectin is downregulated by androgens (36), and that the decline in adiponectin level during puberty in healthy lean males is inversely associated with serum testosterone (32). Interestingly, despite the substantially higher testosterone level in Lz-treated pubertal boys, adiponectin declined similarly in Lz- and Pl-treated pubertal boys during the treatment. Since adiponectin secretion is lower in obese children and adolescents than in their lean peers of corresponding age and pubertal stage (32), this may be explained by the differential changes in body composition in the two groups: the decrease in relative FM may have protected the Lz-treated pubertal boys from a more profound testosterone-induced decline in adiponectin concentration. In theory, Lz-induced hyperandrogenism could predispose obese adolescent boys to hypoadiponectinemia, which has been connected with insulin resistance (37), low HDL-C $(17,32)$, non-alcoholic hepatic steatosis (38), and recently, with signs of early atherosclerosis (18). Therefore, cautious follow-ups of glucose homeostasis and liver function are warranted in obese pubertal boys if treated with an aromatase inhibitor.

In conclusion, in males during puberty, blockade of oestrogen biosynthesis with an aromatase inhibitor and subsequent stimulation of gonadal androgen production adversely affects lipid profile by reducing HDL-C. Therefore, careful follow-up of lipid profile is necessary in pubertal boys receiving aromatase inhibitor treatment. The treatment does not appear to influence insulin sensitivity adversely in lean subjects.

\section{Acknowledgements}

This study was supported by the Foundation for Paediatric Research, Helsinki, Finland.

\section{References}

1 Morrison JA, Laskarzewski PM, Rauh JL, Brookman R, Mellies M, Frazer M, Khoury P, deGroot I, Kelly K \& Glueck CJ. Lipids, lipoproteins, and sexual maturation during adolescence: the Princeton maturation study. Metabolism 197928 641-649.

2 Amiel SA, Sherwin RS, Simonson DC, Lauritano AA \& Tamborlane WV. Impaired insulin action in puberty. A contributing factor to poor glycemic control in adolescents with diabetes. New England Journal of Medicine 1986315 215-219.

3 Cook JS, Hoffman RP, Stene MA \& Hansen JR. Effects of maturational stage on insulin sensitivity during puberty. Journal of Clinical Endocrinology and Metabolism 199377 725-730.

4 Morrison JA, Barton BA, Biro FM \& Sprecher DL. Sex hormones and the changes in adolescent male lipids: longitudinal studies in a biracial cohort. Journal of Pediatrics 2003142 637-642.

5 Moran A, Jacobs DR, Jr, Steinberger J, Cohen P, Hong CP, Prineas R \& Sinaiko AR. Association between the insulin resistance of puberty and the insulin-like growth factor-I/growth hormone axis. Journal of Clinical Endocrinology and Metabolism $2002874817-4820$.

6 Leschek EW, Jones J, Barnes KM, Hill SC \& Cutler GB, Jr. 6-Year results of spironolactone and testolactone treatment of familial male-limited precocious puberty with addition of deslorelin after central puberty onset. Journal of Clinical Endocrinology and Metabolism 199984 575-178.

7 Merke DP, Keil MF, Jones JV, Fields J, Hill S \& Cutler GB, Jr. Flutamide, testolactone, and reduced hydrocortisone dose maintain normal growth velocity and bone maturation despite elevated androgen levels in children with congenital adrenal hyperplasia. Journal of Clinical Endocrinology and Metabolism 200085 1114-1120.

8 Feuillan PP, Jones J \& Cutler GB, Jr. Long-term testolactone therapy for precocious puberty in girls with the McCune-Albright syndrome. Journal of Clinical Endocrinology and Metabolism 1993 77 647-651. 
9 Wickman S, Sipila I, Ankarberg-Lindgren C, Norjavaara E \& Dunkel L. A specific aromatase inhibitor and potential increase in adult height in boys with delayed puberty: a randomised controlled trial. Lancet 2001357 1743-1748.

10 Hero M, Norjavaara E \& Dunkel L. Inhibition of estrogen biosynthesis with a potent aromatase inhibitor increases predicted adult height in boys with idiopathic short stature: a randomized controlled trial. Journal of Clinical Endocrinology and Metabolism $2005906396-6402$.

11 Wickman S \& Dunkel L. Inhibition of P450 aromatase enhances gonadotropin secretion in early and midpubertal boys: evidence for a pituitary site of action of endogenous E. Journal of Clinical Endocrinology and Metabolism $2001 \mathbf{8 6} 4887-4894$.

12 Wickman S, Saukkonen T \& Dunkel L. The role of sex steroids in the regulation of insulin sensitivity and serum lipid concentrations during male puberty: a prospective study with a P450-aromatase inhibitor. European Journal of Endocrinology 2002146 339-346.

13 Morishima A, Grumbach MM, Simpson ER, Fisher C \& Oin K. Aromatase deficiency in male and female siblings caused by a novel mutation and the physiological role of estrogens. Journal of Clinical Endocrinology and Metabolism $1995803689-3698$.

14 Herrmann BL, Saller B, Janssen OE, Gocke P, Bockisch A, Sperling H, Mann K \& Broecker M. Impact of estrogen replacement therapy in a male with congenital aromatase deficiency caused by a novel mutation in the CYP19 gene. Journal of Clinical Endocrinology and Metabolism 200287 5476-5484.

15 Maffei L, Murata Y, Rochira V, Tubert G, Aranda C, Vazquez M, Clyne CD, Davis S, Simpson ER \& Carani C. Dysmetabolic syndrome in a man with a novel mutation of the aromatase gene: effects of testosterone, alendronate, and estradiol treatment. Journal of Clinical Endocrinology and Metabolism 2004 89 61-70.

16 Yamauchi T, Kamon J, Waki H, Terauchi Y, Kubota N, Hara K, Mori Y, Ide T, Murakami K, Tsuboyama-Kasaoka N, Ezaki O, Akanuma Y, Gavrilova O, Vinson C, Reitman ML, Kagechika H, Shudo K, Yoda M, Nakano Y, Tobe K, Nagai R, Kimura S, Tomita M, Froguel P \& Kadowaki T. The fat-derived hormone adiponectin reverses insulin resistance associated with both lipoatrophy and obesity. Nature Medicine 20017 941-946.

17 Cnop M, Havel PJ, Utzschneider KM, Carr DB, Sinha MK, Boyko EJ, Retzlaff BM, Knopp RH, Brunzell JD \& Kahn SE. Relationship of adiponectin to body fat distribution, insulin sensitivity and plasma lipoproteins: evidence for independent roles of age and sex. Diabetologia 200346 459-469.

18 Pilz S, Horejsi R, Moller R, Almer G, Scharnagl H, Stojakovic T, Dimitrova R, Weihrauch G, Borkenstein M, Maerz W, Schauenstein K \& Mangge H. Early atherosclerosis in obese juveniles is associated with low serum levels of adiponectin. Journal of Clinical Endocrinology and Metabolism $2005904792-4796$.

19 Tanner J. Growth at Adolescence. 2nd edn. Oxford, UK: Blackwell Scientific Publications, 1962.

20 Anyan WR Jr. Adolescent Medicine in Primary Care, pp 135-138. New York: John Wiley \& Sons, 1978.

21 Matthews DR, Hosker JP, Rudenski AS, Naylor BA, Treacher DF \& Turner RC. Homeostasis model assessment: insulin resistance and beta-cell function from fasting plasma glucose and insulin concentrations in man. Diabetologia $1985 \mathbf{2 8} 412-419$.

22 Ankarberg C \& Norjavaara E. Diurnal rhythm of testosterone secretion before and throughout puberty in healthy girls: correlation with 17 beta-estradiol and dehydroepiandrosterone sulfate. Journal of Clinical Endocrinology and Metabolism 199984 975-984.

23 Norjavaara E, Ankarberg C \& Albertsson-Wikland K. Diurnal rhythm of 17 beta-estradiol secretion throughout pubertal development in healthy girls: evaluation by a sensitive radioimmunoassay. Journal of Clinical Endocrinology and Metabolism $1996814095-4102$.

24 Friedewald WT, Levy RI, Fredrickson DS \& Halperin M. Estimation of the concentration of low-density lipoprotein cholesterol in plasma, without use of the preparative ultracentrifuge clofibrate in ischemic heart disease. Clinical Chemistry 197218 499-502.

25 Mauras N, O’Brien KO, Klein KO \& Hayes V. Estrogen suppression in males: metabolic effects. Journal of Clinical Endocrinology and Metabolism 200085 2370-2377.

26 Mauras N, Welch S, Rini A \& Klein KO. An open label 12-month pilot trial on the effects of the aromatase inhibitor anastrozole in growth hormone $(\mathrm{GH})$-treated $\mathrm{GH}$ deficient adolescent boys. Journal of Pediatric Endocrinology and Metabolism 200417 1597-1606.

27 Geisler J, Haynes B, Anker G, Dowsett M \& Lonning PE. Influence of letrozole and anastrozole on total body aromatization and plasma estrogen levels in postmenopausal breast cancer patients evaluated in a randomized, cross-over study. Journal of Clinical Oncology 200220 751-757.

28 Tikkanen MJ \& Nikkila EA. Regulation of hepatic lipase and serum lipoproteins by sex steroids. American Heart Journal 1987113 562-567.

29 Sorva R, Kuusi T, Dunkel L \& Taskinen MR. Effects of endogenous sex steroids on serum lipoproteins and postheparin plasma lipolytic enzymes. Journal of Clinical Endocrinology and Metabolism $198866408-413$.

30 Tschritter O, Fritsche A, Thamer C, Haap M, Shirkavand F, Rahe S, Staiger H, Maerker E, Staiger H, Maerker E, Haring H \& Stumvoll M. Plasma adiponectin concentrations predict insulin sensitivity of both glucose and lipid metabolism. Diabetes 200352 239-243.

31 Zietz B, Herfarth H, Paul G, Ehling A, Muller-Ladner U, Scholmerich J \& Schaffler A. Adiponectin represents an independent cardiovascular risk factor predicting serum HDL-cholesterol levels in type 2 diabetes. FEBS Letters $2003 \mathbf{5 4 5} 103-104$.

32 Bottner A, Kratzsch J, Muller G, Kapellen TM, Bluher S, Keller E, Bluher M \& Kiess W. Gender differences of adiponectin levels develop during the progression of puberty and are related to serum androgen levels. Journal of Clinical Endocrinology Metabolism 2004 89 4053-4061.

33 Schneider JG, von Eynatten M, Schiekofer S, Nawroth PP \& Dugi KA. Low plasma adiponectin levels are associated with increased hepatic lipase activity in vivo. Diabetes Care $2005 \mathbf{2 8} 2181-2186$.

34 Zmunda JM, Thompson PD, Dickenson R \& Bausserman LL. Testosterone decreases lipoprotein(a) in men. American Journal of Cardiology 199677 1244-1247.

35 Danesh J, Collins R \& Peto R. Lipoprotein(a) and coronary heart disease. Meta-analysis of prospective studies. Circulation 2000102 1082-1085

36 Lanfranco F, Zitzmann M, Simoni M \& Nieschlag E. Serum adiponectin levels in hypogonadal males: influence of testosterone replacement therapy. Clinical Endocrinology $200460500-507$.

37 Reinehr T, Roth C, Menke T \& Andler W. Adiponectin before and after weight loss in obese children. Journal of Clinical Endocrinology and Metabolism $2004893790-3794$.

38 Targher G, Bertolini L \& Zenari L. Hypoadiponectinemia is closely associated with nonalcoholic hepatic steatosis in obese subjects. Diabetes Care 200427 2085-2086.

Received 8 March 2006

Accepted 8 June 2006 